\title{
Improving Confidence and Practical Skills in Plastic Surgery for Medical Students and Junior Doctors: A One-Day Session
}

\author{
Billy Ching Leung*, Anna De Leo, Roba Khundkar, Nelson Leung, Alistair Reed, Lucy Cogswell \\ Department of Plastic and Reconstructive Surgery, John Radcliffe Hospital, Oxford University Hospital NHS Trust, Oxford, UK \\ Email: *billychingleung@gmail.com
}

How to cite this paper: Leung, B.C., De Leo, A., Khundkar, R., Leung, N., Reed, A. and Cogswell, L. (2016) Improving Confidence and Practical Skills in Plastic Surgery for Medical Students and Junior Doctors: A One-Day Session. Surgical Science, 7, 433442.

http://dx.doi.org/10.4236/ss.2016.79059

Received: August 18, 2016

Accepted: September 19, 2016

Published: September 26, 2016

Copyright $\odot 2016$ by authors and Scientific Research Publishing Inc. This work is licensed under the Creative Commons Attribution International License (CC BY 4.0).

http://creativecommons.org/licenses/by/4.0/ (c) (i) Open Access

\begin{abstract}
Objectives: The current medical school curriculum offers scant exposure to plastic surgery. Medical students and junior doctors are often inadequately prepared for inhospital placements which may impact on patient safety and quality of care. We aimed to deliver a one-day "hands-on" practical session on basic surgical skills and principles in plastic surgery, and to assess its effectiveness at improving confidence to the specialty among junior trainees. Methods: The one-day practical session covered knot tying, suturing, LA administration, skin lesion excision and local flaps, with short concurrent lectures on principles of plastic surgery. Self-assessment confidence scores were recorded before and after the course on seven domains: a) knowledge: wound assessment and management, suture selection, and concepts of flaps; b) practical skills: Handling of surgical instrument, suturing, LA administration, and skin lesion excision. Results: Thirty-five participants attended: Six core surgical trainees, fifteen foundation doctors, and fourteen medical students. The overall course satisfaction was 9.13/10. Self-assessment confidence scores in all domains significantly improved after the course $(p<0.0001)$. Suturing $(58.6 \%)$ and skin lesion excision (74.5\%) demonstrated the largest improvement. Qualitative feedback was extremely positive. Conclusions: The hands-on nature of the course offered participants great opportunities to acquire the necessary confidence and practical skills required for in-hospital placements. It is vital for students and doctors interested in a career in plastic surgery to prepare adequately for this diverse specialty, which is covered in limited depth in medical school. The need for more practical skills courses on plastic surgery for this demographic is intuitive.
\end{abstract}

\section{Keywords}

Course, Practical Skills, Plastic Surgery, Undergraduate, Curriculum 


\section{Introduction}

Plastic surgery is a specialty that involves the restoration of form and function, and the reconstruction of tissue defects; it covers a wide spectrum of conditions and pathologies including burns, hand traumas, soft tissue defects, skin oncology, breast reconstructions, and cleft and craniofacial deformities. The historic meaning of "plastic surgery" originates from the Greek word plastikos, which translates to "to mold" or "to form" [1]. The foundations of plastic surgery are based upon anatomical and physiological principles and surgical precision and finesse, which are essential components for medical students and junior doctors to appreciate prior to plastic surgery hospital placements.

Undergraduate teaching of plastic surgery has declined in recent years; in the 1980s, $78 \%$ of medical schools formally taught plastic surgery, this fell to $21 \%$ and $13 \%$, in the 1990s and 2000s respectively [2]-[4]. The curriculum evolved to accommodate the modernisation of medical education which focused on a broader syllabus including patient-centred care and public health, but at the expense of certain specialty components [5]-[7]. Consequently, junior doctors and medical students harbour a number of misconceptions about the specialty thus hampering the opportunity to make informed career choices; this is particularly poignant given the current trend to encourage early specialisation [7]-[9]. At present, the exposure to plastic surgery is predominantly through self-selected modules (SSC) and elective studies for medical students, and "taster-weeks" for junior doctors [10].

More importantly, junior doctors are often insufficiently prepared for plastic surgery rotations during foundation or core surgical training, and this may lead to suboptimal patient care and adverse clinical outcomes. A parallel comparison can be made with the undergraduate teaching of anatomy in the past decades. The Medical Defense Union (MDU) reported a 7-fold increase in medical errors related to poor anatomical knowledge in doctors between 1995 and 2000 [11]. This highlighted that adequate undergraduate training is paramount for the delivery of safe patient care.

The benefit of one-day practical skills courses on plastic surgery has been documented previously by two national courses, both displayed a significant improvement in delegate post-course knowledge and practical skills; delivered by The British Association of Plastic and Reconstructive Surgeons (BAPRAS Undergraduate Day) and St Georges Hospital [7] [12]. Other courses include: MY Plastic Surgery Course (two-day course in Wakefield for students and doctors), Facial Plastic Surgery Course (three-day course in London for doctors), and specialty courses by The Royal College of Surgeons of England (RCSeng), such as Specialty Skills in Plastic Surgery [13].

There are encouraging numbers of courses on plastic surgery for medical students and junior doctors, however, considering its popularly and scant undergraduate exposure, we propose a need for more. We aimed to deliver a one-day "hands-on" practice session on basic surgical skills and principles in plastic surgery for medical students and junior doctors, and assess its effectiveness at improving their confidence to the specialty. 


\section{Methods}

We delivered a one-day practical session titled: Basic Surgical Skills and Principles in Plastic Surgery for Junior Doctors and Medical Students, on $27^{\text {th }}$ January 2015 at John Radcliffe Hospital, Oxford. No fee was charged for attendance. Invitations were emailed to medical students at the University of Oxford, St George's University of London, and King's College London, foundation doctors, and core surgical trainees training in the Thames Valley Deanery (Oxford University Hospital NHS Trust, Buckinghamshire Healthcare NHS Trust, Royal Berkshire NHS Foundation Trust, Heatherwood and Wexham Park Hospital NHS Trust, and Milton Keynes General NHS Trust).

The course was divided into morning and afternoon sessions. Each session contained short lectures and practical skills stations (Figures 1-4). Short lectures (10 minutes per lecture) were delivered to all delegates in the clinical skills lab via PowerPoint presentations and videos, by consultants, specialist registrars and core surgical trainees. Practical skills stations were divided into five individual groups depending on grade: Core
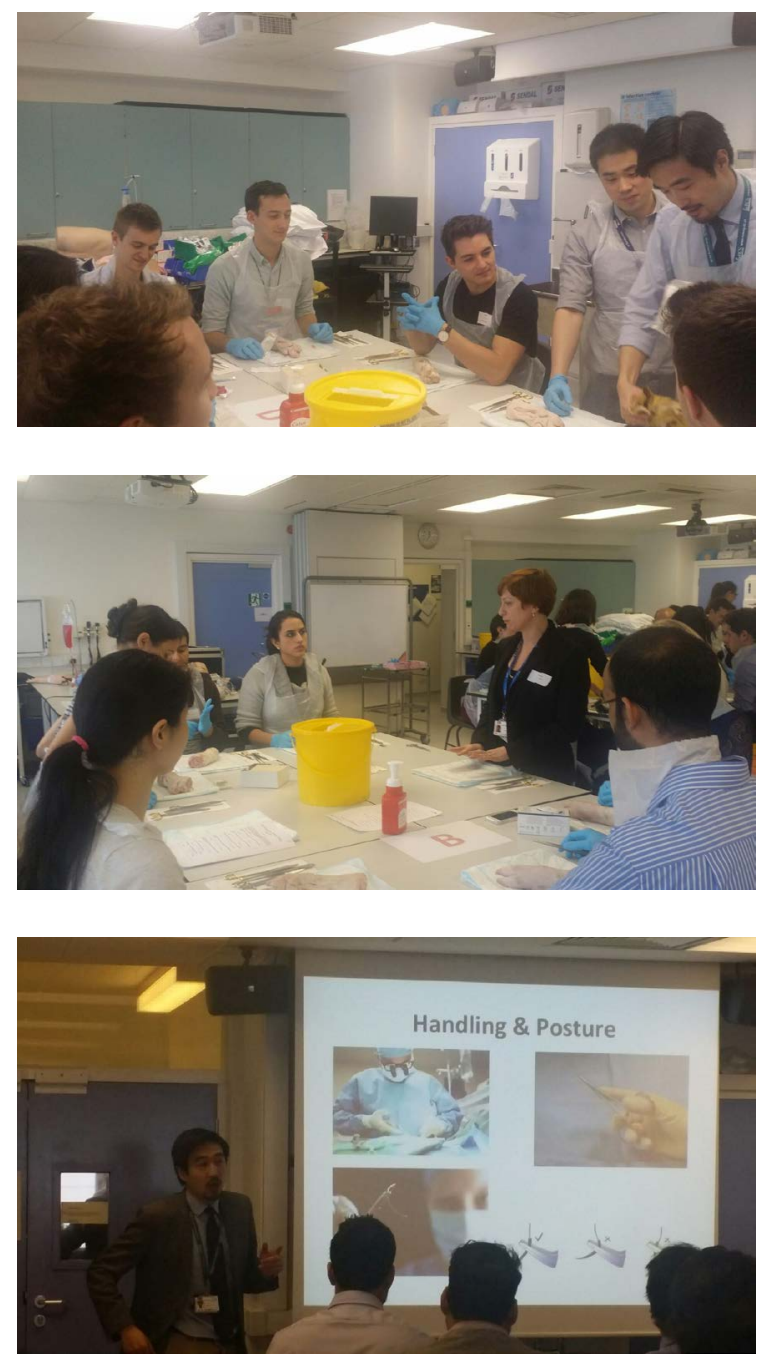

Figure 1. Practical sessions (top \& middle); short lectures (bottom). 


\begin{tabular}{|c|c|c|c|c|}
\cline { 2 - 5 } \multicolumn{1}{c|}{} & Pre-course mean (SEM) & Post-course mean (SEM) & \% improvement & \multicolumn{1}{c|}{$p$-value } \\
\hline Knowledge (Av. Score) & $5.13(2.11)$ & $7.55(1.56)$ & $41 \%$ & $<0.0001$ \\
\hline Wound assesement \& treatment & $5.06(2.49)$ & $7.34(1.82)$ & $45.1 \%$ & $<0.0001$ \\
Suture selection & $5.31(2.10)$ & $7.72(1.37)$ & $45.4 \%$ & $<0.0001$ \\
Concept of flaps & $5.03(2.02)$ & $7.47(1.48)$ & $32.7 \%$ & $<0.0001$ \\
\hline
\end{tabular}

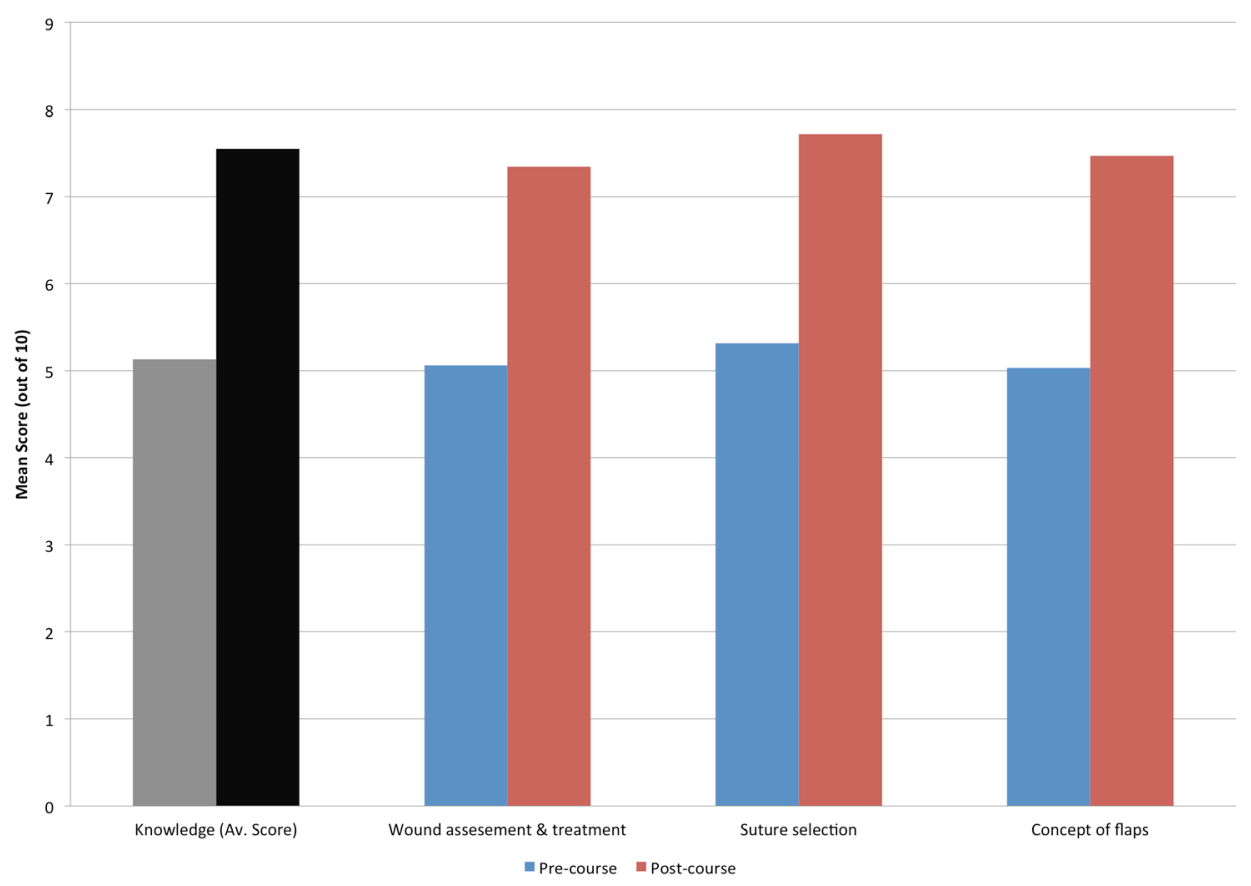

Figure 2. Self-assessment confidence scores for "knowledge"-table (top): Mean, standard error to mean (SEM), percentage improvement and $p$-value, graph (bottom): Mean scores before and after the course (grey and black bars: Average scores of all knowledge domains).

surgical trainees year 1 (CT1), foundation year 2 doctors (FY2), foundation year 1 doctors (FY1), senior medical students (MBBS 4-6), and junior medical students (MBBS 1-3). All groups were tutored by a consultant or a specialist registrar, except for the pre-clinical medical student group, which was led by a core surgical trainee. The ratio of faulty-to-student was 1:6 per group.

The morning session consisted of short lectures on: introduction to plastic surgery, principles of plastic surgery, assessment and management of wounds, suturing materials and products, and general handling of surgical instruments. The practical sessions included: knot tying (basic square knot, 1-hand tie and 2-hand tie) and suturing (simple interrupted, horizontal and vertical mattress, subcuticular, deep dermal, running, running locking and corner stitch). The afternoon session consisted of short lectures on: local anaesthetic administration, excision of skin lesions, and basic concepts of local flaps. The practical session included: local anaesthetic dosing and administration, excision of skin lesions (with closure of wounds using skills learnt from the morning session), and construction of local flaps (advancement, rotational, transposition, and Z-plasties). Pig trotters were used in the practical sessions for suturing, LA administration, skin lesion excision, and local flap construction. Pig trotters were used in the prac- 


\begin{tabular}{|c|c|c|c|c|}
\cline { 2 - 5 } \multicolumn{1}{c|}{} & Pre-course mean (SEM) & Post-course mean (SEM) & \% improvement & \multicolumn{1}{c|}{$p$-value } \\
\hline Practical Skills (Av. Score) & $5.15(2.25)$ & $7.68(1.54)$ & $51 \%$ & $<0.0001$ \\
\hline Handling of instruments & $6.38(1.93)$ & $7.97(1.51)$ & $58.9 \%$ & $<0.0001$ \\
Suturing & $4.81(2.22)$ & $7.63(1.39)$ & $48.7 \%$ & $<0.0001$ \\
Local anaesthetics & $5.13(2.54)$ & $7.63(1.84)$ & $74.5 \%$ & $<0.0001$ \\
Skin lesion excision & $4.28(2.30)$ & $7.47(1.54)$ & & $<0.0001$ \\
\hline
\end{tabular}

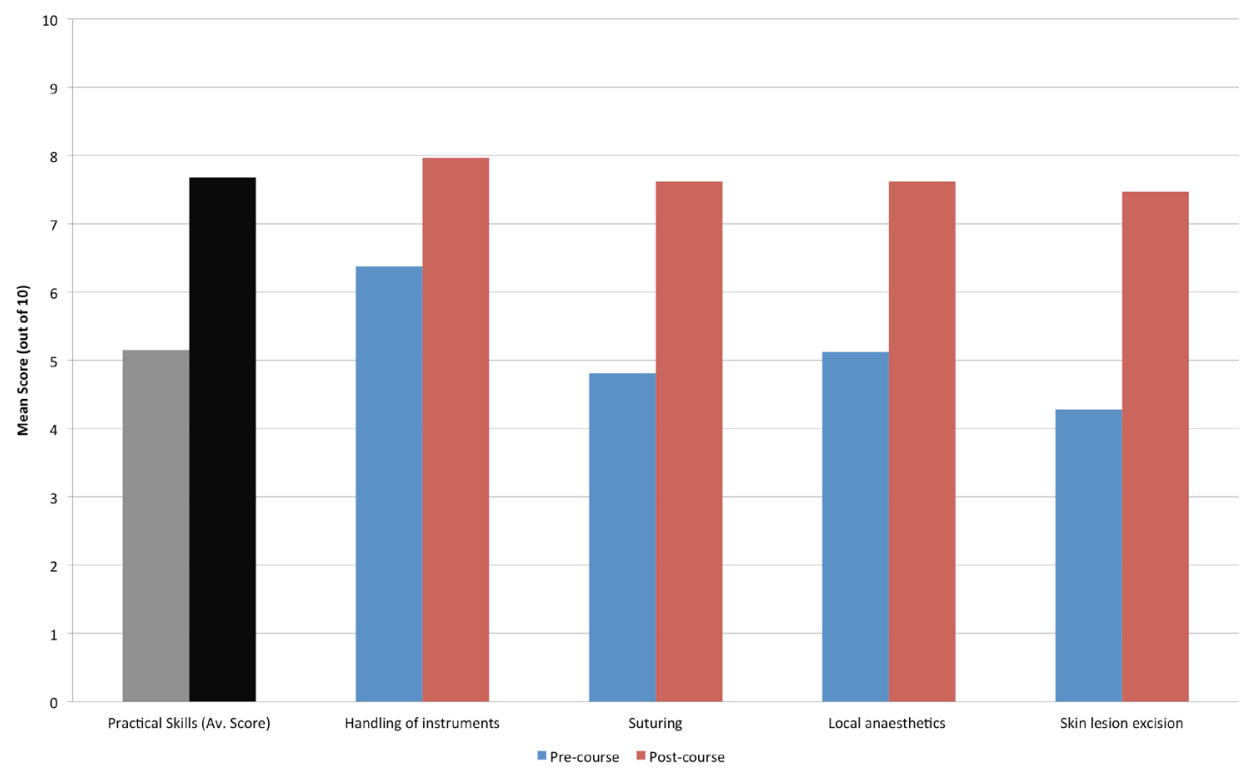

Figure 3. Self-assessment confidence scores for "practical skills"-table (top): Mean, standard error to mean (SEM), percentage improvement and $p$-value, graph (bottom): Mean scores before and after the course (grey and black bars: Average scores of all knowledge domains).

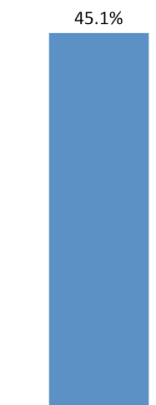

Wound assesement \& treatment

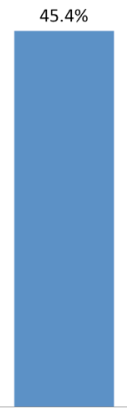

Suture selection

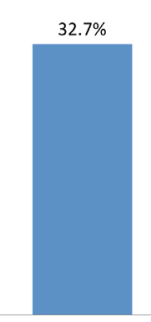

Concept of flaps

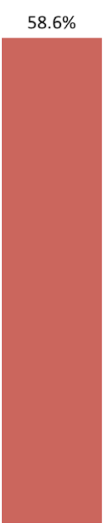

Suturing

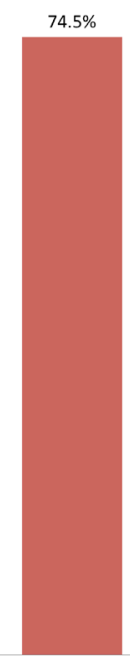

Skin lesion excision

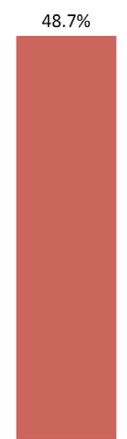

Local anaesthetics

Figure 4. Mean percentage improvement after the course (blue $=$ knowledge; red $=$ practical skills).

tical sessions for suturing, LA administration, skin lesion excision, and local flap construction (approved by hospital trust).

A questionnaire was used to record the self-assessment confidence scores before and 
after the course using a modified Likert scale (range 1 to $10 ; 1=$ low, $5=$ moderate, $10=$ high) on seven knowledge and practical skills domains: a) knowledge domains: wound assessment and management, suture selection, and concept of flaps; and b) practical skills domains: handling of surgical instruments, suturing, LA administration, and skin lesion excision. The scores were expressed as mean values and the standard error to the mean (SEM), and a paired $t$ test was used for comparative statistical analysis before and after the course. Statistical significance was set as $p<0.05$.

The questionnaire also included: a) what is your desired career interest; b) overall satisfaction rate (out of 10); and c) qualitative questions that included: why have you attended the course? what useful skills have you learnt today? and how can we improve the course?

\section{Results}

A total of 134 medical students and doctors replied and expressed interest: eight CT1 (5.9\%), fifteen FY2 (11\%), twelve FY1 (9\%) and 98 MBBS students (73.1\%). 35 places were available and were offered as a first come first served basis, with priority given to doctors. 35 participants attended: six CT1 (16.7\%), ten FY2 (28.6\%), five FY1 (3.7\%), six MBBS year 5 (14.3\%), two MBBS year 4 (5.7\%), four MBBS year 3 (11.4\%), and two MBBS year 2 (5.7\%). All 35 questionnaires were completed. 33 delegates intended a career in surgery (specialty not specified in the questionnaire) and two delegates (both MBBS year 2) were unsure. The overall course satisfaction score was 9.13/10.

\section{Knowledge domains}

All knowledge domains demonstrated significant improvement in self-assessment confidence scores after the course $(p<0.0001)$ : wound assessment and management (45\%: pre-course mean 5.06 (SEM 2.49), post-course mean 7.45 (SEM 1.82)); suture selection (45.6\%: pre 5.31 (2.10), post 7.72 (1.37)); concept of flaps (32\%: pre 5.03 (2.02), post $7.47(1.48)$ ). The average improvement was $41 \%$ (pre 5.13 (2.11), post $7.55(1.56)$ ).

\section{Practical skills domains}

All practical skills domains demonstrated significant improvement in self-assessment confidence scores after the course $(p<0.0001)$ : handling of surgical instruments (24.9\%: pre-course mean 6.38 (SEM 1.93), and post-course 7.97 (SEM 1.37)); suturing (58.6\%: pre 4.81 (2.22), post 7.63 (1.39)); LA administration (48.7\%: pre 5.13 (2.54), post $7.63(1.84)$ ); skin lesion excision (74.5\%: pre $4.28(2.30)$, post $7.47(1.54))$. The average improvement was $51.2 \%$ (pre 5.15 (2.25), post $7.68(1.54)$ ).

Qualitative feedback

The qualitative feedback was extremely positive and most delegates would highly recommend the course to other colleagues. The most common reasons for attendance were: "to learn more about plastic surgery due to career interest", "to prepare for hospital placements in plastic surgery", and "the lack of plastic surgery teaching in medical schoop". The most useful things the delegates found were: "understanding of the principles of plastic surgery", "able to practice suturing techniques and receive feedback from consultants and registrars", and "understanding the concepts of local flaps and ability 
to create them during the course". Other comments include: "good balance of lectures and practical skills sessions", "adequate time to practice practical skills and ask questions", and "good faculty-to-student ratios".

\section{Discussion}

Our one-day practical skills session stimulated abundant interests from 134 medical students to junior doctors across Oxford and London. The highest interest was from medical students (98/134-73.1\%), which may be due partly to their relative accessibility, but also to the potential deficiency in the exposure to plastic surgery in the undergraduate curriculum [2]-[4]. The second largest group was FY2 (15/134-11\%). Doctors at this stage often become senior house officers ( $\mathrm{SHO}$ ) in plastic surgery with limited prior experience, thus, this course may offer the Foundation Year 2 doctors the opportunity to acquire the skills required to perform "SHO" duties, such as wound assessments and suturing they may not have the chance to develop in undergraduate training and hospital placements. Finally, eight CT1 doctors expressed interest, which accounted for $36.4 \%$ (8/22) of the whole cohort of CT1 doctors in Thames Valley, highlighting the popularity of this specialty and the necessity of the course.

Students" confidence in all knowledge and practical skills domains showed significant improvement after the course $(p<0.0001)$. Firstly, the overall improvement of practical skills domains was $51 \%$, with "skin lesion excision" and "suturing" demonstrating the highest improvement out of all domains, $74.5 \%$ and $58.6 \%$, respectively. "LA administration" also significantly improved by $48.7 \%$. This highlighted the efficacy of the course at improving students" confidence in practical skills, which is essential for the delivery and provision of safe patient care. The hands-on nature of the course and the targeted selection of the taught domains, offered participants great opportunities to acquire the necessary confidence and practical skills required for in-hospital placements. This is particularly important in an era where undergraduate exposure to plastic surgery has reduced; students and doctors are often inadequately prepared for surgical rotations [2]-[4]. An alarming comparison can be made with the teaching of anatomy in past decades that lead to a detrimental impact on patient safety due to its decline. A recent study published by the MDU revealed 32\% of general and vascular surgery claims were related "damage to underlying structures" due to poor anatomical knowledge [11] [14]-[17].

Interestingly, of all the domains, "handling of surgical instruments" demonstrated the lowest post-course improvement (24.9\%) but highest pre-course mean score of $6.38 / 10$. This highlighted the adequacy of general surgical concepts and knowledge compared to the specialty concepts. The knowledge domains of "wound assessment and management", "suture selection", and "concepts of flaps" all demonstrated significant improvement of $45.06 \%, 45.59 \%$ and $32.55 \%$, respectively. This further supported the efficacy and usefulness of concurrent short lectures delivered during the course.

Qualitative feedback was extremely positive. Delegates were highly satisfied with the time provided for practical skills sessions; it allowed junior delegates to practice and 
repeat taught skills, and senior delegates to fine-tune their skills and seek more advanced practical skills outside the course curriculum. The high faculty-to-student ratio (1:6) and consultant and senior registrar-led nature of the course greatly benefited the delegates. Students complimented that all demands and questions were met and answered.

Some core surgical trainees expressed the need for a greater emphasis on more specialist skills, such as local flaps and tendon repair, rather than basic surgical skills, such as knot tying and suturing. A future aim is to address this in our next course by enquiring about the specific needs of more senior delegates prior to the course, or alternatively, by delivering an independent practical skills course for more senior delegates. Surgical courses aimed at more experienced and skilled delegates include MY Plastic Surgery Course (two-day course for students and doctors), Facial Plastic Surgery Course (three-day course for doctors), and the specialty courses by the RCSeng (Specialty Skills in Plastic Surgery, Emergency Skills in Maxillofacial Surgery, and various regional anatomy courses) [13]. These courses are often expensive, costing up to $£ 300$ 400 per session, which may not be feasible to all medical students and junior doctors.

There are emerging numbers of plastic surgery courses for both medical students and junior doctors, with published articles highlighting the benefit of one-day courses in enhancing undergraduates perception, knowledge and skills in plastic surgery [7] [12]. However, considering its reduced undergraduate exposure and popularity as a career choice, the number of plastic surgery courses is still limited. Furthermore, an article published by the RCSeng highlighted that stimulation training, such as skills courses, is an essential training tool for surgical trainees to compensate for the significant decline in operative training time since the introduction of the European Working Time Directive (EWTD) [18] [19]. Thus, the need for more surgical skills courses is intuitive.

\section{Limitations}

There were several limitations to our study. There were several useful domains that we were unable to cover due to time and resource restrains, such as burns management, hand fracture fixation and tendon repair. The questionnaire did not specifically ask delegates their perception and experience of undergraduate plastic surgery training, and their overall confidence in starting in a plastic surgery rotation.

\section{Future considerations}

We aim to deliver the course on a regular basis, biannually, at the John Radcliffe Hospital, and potentially introduce it as a formal component of the medical school curriculum at the University of Oxford. Future courses may be organised independently for medical students, foundation doctors and core surgical trainees, as this would allow us to accurately tailor the level of knowledge and skills delivered, which would maximise the efficacy of the course.

Overall, despite the decline in undergraduate exposure to plastic surgery, the specialty remains one of the most popular and competitive in the UK and worldwide, and committed individuals to plastic surgery often seek exposure beyond their standard curriculum [20]. We believe that the course will significantly benefit junior doctors and 
medical students interested in plastic surgery, and ultimately patient care. By defining a targeted course objective that prepares delegates for in-hospital rotations, this will optimise their application of the simulated skills onto clinical practice.

\section{Conclusion}

The decline in undergraduate exposure in plastic surgery in recent decades has potential impact on the knowledge and practical abilities of medical students and junior doctors, which may have clinical implications. We organised a one-day plastic surgery skills course for students and doctors, and assessed its efficacy in improving their knowledge and practical skills in the specialty. The course received excellent feedback, with all domains in knowledge (e.g. wound assessment and management) and practical skills (e.g. skin lesion excision and suturing), demonstrating significant improvement after the course. Delegates were highly satisfied with the practical-focused nature of the course, and found the concurrent lectures useful in supplementing the theory behind the practical skills. The consultant and senior registrar-led nature of the course, together with high faculty-to-student ratio, were warmly received and complimented. The course will be held biannually at the John Radcliffe Hospital and potentially integrated into the medical school curriculum at the University of Oxford. Similar courses are available, but are often costly and infrequent. We believe our course will provide medical students and junior doctors the necessary basic knowledge and practical skills required for plastic surgery rotations, and consequently enhance the quality of care delivered alongside patient safety.

\section{References}

[1] Kim, J. (2014) Plastic Surgery. Encyclopaedia Britannica.

[2] Rowsell, A.R. (1986) The Place of Plastic Surgery in the Undergraduate Surgical Curriculum. British Journal of Plastic Surgery, 39, 241-243. http://dx.doi.org/10.1016/0007-1226(86)90090-1

[3] Porter, J.M., Rayner, C.R. and Fenton, O.M. (1992) Teaching Plastic Surgery to Medical Students. Medical Education, 26, 42-47.

http://dx.doi.org/10.1111/j.1365-2923.1992.tb00121.x

[4] Rees-Lee, J.E. and Lee, S. (2008) Reaching Our Successors: The Trend for Early Specialisation and the Potential Effect on Recruitment to Our Speciality. Journal of Plastic Reconstructive and Aesthetic Surgery, 61, 1135-1138.

[5] Turney, B.W. (2007) Anatomy in a Modern Medical Curriculum. Annals of The Royal College of Surgeons of England, 89, 104-107. http://dx.doi.org/10.1308/003588407X168244

[6] Ramsden, P. (2003) Approaches to Learning (Chapter 4). In: Ramdsen, P., Ed., Learning to Teach in Higher Education. 2nd Edition, Approaches to Learning, Routledge Falmer, Oxford, 39-61.

[7] Khatib, M., et al. (2014) Plastic Surgery Undergraduate Training: How a Single Local Event Can Inspire and Educate Medical Students. Annals of Plastic Surgery, 75, 208-212.

[8] Parikh, A.R., Clarke, A. and Butler, P.E. (2006) Plastic Surgery and the Undergraduate Medical School Curriculum. Medical Education, 40, 476-477. http://dx.doi.org/10.1111/j.1365-2929.2006.02425.x 
[9] Taylor, I., Reed, M.F., Kingsnorth, A.N., Carlson, G.L. and Leaper, D.J. (2005) Surgery in the Undergraduate Curriculum. Annals of The Royal College of Surgeons of England, 87, 138-139.

[10] Ng, Z.Y. (2012) Developing Undergraduate Interest in Plastic Surgery. Journal of Plastic Reconstructive and Aesthetic Surgery, 65, 691-692.

http://dx.doi.org/10.1308/147363505X39239

[11] Ellis, H. (2002) Medico-Legal Litigation and Its Links with Surgical Anatomy. Surgery, 20, 1-2. http://dx.doi.org/10.1383/surg.20.8.0.14518

[12] Davis, C.R., et al. (2010) How to Improve Plastic Surgery Knowledge, Skills and Career Interest in Undergraduates in One Day. Journal of Plastic Reconstructive and Aesthetic Surgery, 63, 1677-1681. http://dx.doi.org/10.1016/j.bjps.2009.10.023

[13] Jalali, M., et al. (2011) Skills Courses in Plastic Surgery: Impact of Enhancing Confidence, Skills and Understanding amongst Junior Doctors. Journal of Plastic Reconstructive and Aesthetic Surgery, 64, 976-978. http://dx.doi.org/10.1016/j.bjps.2010.10.025

[14] McHanwell, S.M., et al. (2007) A Core Syllabus in Anatomy for Medical Students-Adding common Sense to Need to Know. European Journal of Anatomy, 11, 3-18.

[15] Waterston, S.W. and Stewart, I.J. (2005) Survey of Clinicians' Attitudes to the Anatomical Teaching and Knowledge of Medical Students. Clinical Anatomy, 18, 380-384. http://dx.doi.org/10.1002/ca.20101

[16] McKeown, P.P., et al. (2003) The Impact of Curricular Change on Medical Students' Knowledge of Anatomy. Medical Education, 37, 954-961. http://dx.doi.org/10.1046/j.1365-2923.2003.01670.x

[17] Prince, K.J., et al. (2005) Do Students Have Sufficient Knowledge of Clinical Anatomy? Medical Education, 39, 326-332. http://dx.doi.org/10.1111/j.1365-2929.2005.02096.x

[18] RCSeng (2009) Surgeons Call for Solution on Patient Safety and Future Training as Doctors Hours Are Slashed. RCSeng.

[19] Giles, J.A. (2010) Surgical Training and the European Working Time Directive: The Role of Informal Workplace Learning. International Journal of Surgery, 8, 179-180. http://dx.doi.org/10.1016/j.ijsu.2010.01.011

[20] Jalali, M., et al. (2011) The UK Plastic Surgery Trainee. Journal of Plastic Reconstructive and Aesthetic Surgery, 64, 1716-1717. http://dx.doi.org/10.1016/j.bjps.2011.06.006

\section{Abbreviations}

LA: Local anaesthetics;

RCSeng: Royal College of Surgeons of England;

BAPRAS: British Association of Plastic, Reconstructive, and Aesthetic Surgery;

EWTD: European Working Time Directive 
Submit or recommend next manuscript to SCIRP and we will provide best service for you:

Accepting pre-submission inquiries through Email, Facebook, LinkedIn, Twitter, etc. A wide selection of journals (inclusive of 9 subjects, more than 200 journals)

Providing 24-hour high-quality service

User-friendly online submission system

Fair and swift peer-review system

Efficient typesetting and proofreading procedure

Display of the result of downloads and visits, as well as the number of cited articles

Maximum dissemination of your research work

Submit your manuscript at: http://papersubmission.scirp.org/

Or contact ss@scirp.org 